\title{
Evidence based dentistry research: An overview
}

\author{
Lloyd A. Wallin* \\ 50 East McAndrews Road, Burnsville, Minnesota 55337, USA
}

\begin{abstract}
For Evidence Based Research to become a valid part of an Evidence Based Dentistry Profile, consideration(s) must be given to the fact that dental care tends to be an elective service that is directly related to cost. This set dental care completely apart from medical care.
\end{abstract}

\section{Introduction}

Traditionally, "academia" has been successful in influencing lawmakers about health care reform. Minnesota's Dental Therapist Legislation [1] that was enacted in 2009 is the perfect example. Academia and lawmakers, however, are totally oblivious to what it costs to run a dental practice, and for some unexplained reason, they are striving to keep provider reimbursement levels at a bare minimum.

If the charge of EBD is to help dentists understand the rationale for the things they do and why they do them [2], then EBD "research data" must include cost analysis input from practicing dentists. I am a practicing dentist and the motivation to write this paper is to discuss information that will affect EBD relative to the cost(s) of service(s). Unlike medical care, and I will talk about this in more detail later, dental care remains an elective service and as such, patients will have, and must continue to have the option to choose their dental treatment based on their own priorities.

\section{"Number One" The effect of natural selection on case planning}

Elective cosmetic dentistry remains very influential in the natural selection process of human reproduction in our culture today. This may be viewed as selective genetic engineering in its purest form, or it may be just another form of learned behavior, but it does have a cost effect in dental care. In either case EBD proponents must pay specific attention to effect that the natural selection process plays when making decisions concerning dental care options.

A case in point is within the practice of Orthodontics. In layman's terms, when a patient who has undergone the time, expense and inconvenience of orthodontic treatment for primarily cosmetic purposes, this patient will also select a mate with straight teeth. In today's society, a "beautiful smile" suggests the pathway to popularity and success. This overlooked phenomenon will, in successive generations, ultimately produce offspring that will have much greater orthodontic/cosmetic problems than did their parents, and this will directly affect costs.

\section{"Number Two" The negative cost effect of bundling dental procedures}

A significant part of whatever conclusions are reached with EBD will also be dependent upon the reliability of the data used to support a cost analysis that is based on treatment procedure(s). If the financial data is flawed, so then will be the subsequent EBD research conclusions. As a prime example of flawed financial data in dentistry, one only needs look to the ongoing misrepresentations of data from the American Dental Association's Code Revision Committee ("CRC") in Chicago, Illinois.

Up until 2005, according to Luther [3], "ADA Policy stated that bundling by payers is a potentially fraudulent and abusive practice and is defined as: "The systematic combining of distinct dental procedures by third party payers that results in a reduced benefit for the patient/ beneficiary." This, without question, affects the true cost of dental care.

An excellent example of how the CRC actually promotes "bundling," is the ADA Code descriptor that states: "local anesthesia is usually considered to be part of restorative procedures" [4]. Many dentists throughout the Country routinely itemize and charge separately for local anesthesia using the CDT Code D9215 [5]. When this Code submitted to payers, and because of the CRC descriptor, payers "bundle" the charge submitted for the local anesthesia with the charge submitted for the dental procedure, and the provider is essentially not paid for the local anesthesia unless that cost is allowed to be passed on to the subscriber by the payer. This fosters complete misrepresentation of the true cost of most dental services.

Some are suggesting that the bundling of local anesthesia is "a fraudulent act" when a service is meant to include a charge for local anesthesia and local anesthetic was not used. Because the ADA Code remains unwilling to provide a separate "Code" for the restorative services when a local anesthesia is not used, providers are being misled into fraudulently charging subscribers for a service that has not been used. This actually occurs between 40 and 50 percent of the time on patients who do not choose to use, or need to use, local anesthesia [6].

In simple terms, those patients using dental plans who do not need, or choose to use local anesthesia, are paying for local anesthesia because the bundling of local anesthesia is promoted by the CRC and endorsed

Correspondence to: Lloyd A. Wallin, 50 East McAndrews Road, Burnsville, Minnesota 55337, USA, Tel: 952-892-5050; E-mail: 1loyd@lwallin.com

Received: May 10, 2015; Accepted: June 10, 2015; Published: June 13, 2015 
by the ADA. Therefore, bundling breeds flawed cost data, and all dental services that bundle anything must be re-evaluated. The bundling of local anesthesia is only one example of fraudulent, consumer cost data manipulation that the ADA has allowed to go on for years.

\section{"Number Three" Too many specialists can inflate the cost of care}

The over utilization of "Specialists" and/or the underutilization of "Generalists" for routine oral surgical, endodontic, pediatric and periodontal treatment is making a profound impact on the cost of many dental services. Throughout the nineteen fifties and sixties, dental schools throughout the United States educated students to be able to extract teeth, do root canal treatment, periodontal surgery, as well as traditional restorative dentistry. As the eighties and nineties emerged, the dental schools found it profitable to teach post graduate oral surgery, endodontics and periodontics outside of the traditional four year dental curriculum.

While it is true that some generalists learn oral surgery, etc., as they practice their profession, most generalists, in recent years, have been "conditioned" by academia to refer everything outside basic restorative services, to specialists. This significant change in the patient referral mindset of generalists, has ultimately led to major increases in the overall costs of dental care as follows:

a) Specialists charge more and get paid much more when performing many of the same routine services formerly done by general dentists.

b) Third party payers pay specialists more to do exactly the same services that are and were previously performed by generalists.

c) Publicly funded dental plans also pay more to specialists than to generalist when doing exactly the same dental procedures.

d) The primary motivation for a general dentist to become a specialist is simply because they can get paid more for doing many of the same procedures. This has not only created a shortage of generalists, but the generalists are no longer being effectively taught the same art and science of dentistry as their predecessors.

EBD research must develop a comprehensive cost comparison between competing specialist and generalists. Identical treatment procedures being performed at substantially different costs will not provide reliable data upon which to determine dental treatment based on cost. This, in many respects, is no different from Dental Boards permitting different standards of care for the same procedures depending on the provider.

\section{“Number Four" Specialty boundaries no longer exist}

As competition for patients continues to increase between dentists, specialists are doing more and more procedures formerly done by generalists, just to keep busy. Who would have ever guessed that Oral Surgeons would be making the crowns for implants they placed? Who would have ever thought that Endodontists would be placing the dowel posts into the teeth they did root canals on? Who would have ever dreamed that Periodontists would not only be placing implants, but also doing the oral surgery in preparation for the implant(s)?

If specialists will be able to freely operate out side of their own specialty, does this then increase the cost dentistry? Are specialists forced, because of competition, to take work from generalists? Will third party payers remain willing to pay specialists more to do the same procedures that generalists did? Should generalists and specialists be paid the same?

I asked the Minnesota Board of Dentistry [7] what, if any, problems they are anticipating with regard to dental specialists performing dental procedures that are outside of the "specialty" that they limit their practice to. I was told that they have had no complaints, so far, and that it would depend upon what the Professional Association of each "Specialty" chooses to allow.

Granted these are emerging questions that are surfacing throughout the United States, relative to what dental specialists are allowed to do. If the charge of EBD is to recommend ways to control rising dental costs, and secondarily, reduce the "unnecessary" dental work being done in a sea of Dental Boards who have seemingly lost sight of their own Rules, I submit that EBD proponents must first deal with the guidelines that here-to-fore, they have either not been aware of, or they have chosen to neglect.

\section{"Number Five" The use of legislation to control dental costs}

Self interest groups can seriously manipulate the legislative process that can, in turn, precipitate a negative effect on both the quality and the cost of dental care. Case in point is the Minnesota Dental Therapist Legislation that was passed by Minnesota Lawmakers in 2009, and signed into law by, then, Governor Tim Pawlenty.

In a series of steps that were carefully planed and executed by self interest groups, opposition to the Legislation was completely ignored by the media [8]. The dental therapist legislation was also opposed by all licensed, general dentists [9] in Minnesota, as well as by the entire dental student population at the Minnesota School of Dentistry.

What EBD must learn from what has happened in Minnesota due to the mindset of lawmakers suggesting that "there are not enough dentists," is that when there are too many dentists, they will do work that is not necessarily necessary just to keep busy. Some will, of course, call it "preventive dentistry." EBD must come to understand that when the perceived need for dental care is skewed, so then also will be the perceived cost of providing the care.

\section{Conclusion}

One simply cannot compare the need of dental care to other forms of heath care services, simply because out-of-pocket cost, as well treatment fear, are vital factors in electing to have, or not have, various kinds of dental treatment. Because of this basic human behavior, I submit that literally all of the current "models" and "scientific research" being used to "define evidence based dentistry" are of limited value and must be reevaluated and redone.

The ADA definition of Evidence Based Dentistry, included within the Association's EBD policy statement [10], explicitly addresses the need to incorporate patients' characteristics in treatment planning, including their "treatment needs and preferences," social factors and the dentist's judgment of the patient compliance level with recommended treatments. Expanding the scientific basis for clinical care also will increase patients' access to better information, and could significantly affect the choices they make regarding their oral health care [11].

While the factors of treatment choice based on cost and fear may be interpreted as being included in the American Dental Association's definition of Evidence Based Dentistry: "to incorporate patients' characteristics in treatment planning." The intent if this paper is to 
significantly expand and bring to light those the factors that are elective in nature and fueled by out of pocket cost.

\section{References}

1. Minnesota School of Dentistry (2009)The Minnesota State Legislature enacted legislation supported by the Minnesota School of Dentistry as well as the Officers of the Minnesota Dental Association (not the Membership), to graduate a class of "Dental Therapists" directly from the Minnesota School of Dentistry. A Dental Therapist will be "licensed" to place dental restorations, extract teeth and write prescriptions under a collaborative agreement with a licensed dentist.

2. Evidence-based Dentistry, Ways to integrate EBD in practice, ADA News November 16, 2009, Jennifer Garvin.

3. Luther John R (2005) American Dental Association Interim Associate Executive Director, Dental Practice Director Council on Dental Practice, per letter to Dr. Lloyd A. Wallin, dated August 10.

4. ADA Practical Guide to Dental Procedure Codes; (C2011 - 2012 CDT page 13 American Dental Association.
5. Ibid.

6. Alan Friedel (2010) Committee Meeting Records, American Dental Association Code Revision Committee.

7. Minnesota Board of Dentistry, University park Plaza, 2829 University Ave. SE, Suite 450, Minneapolis, MN 55414-3249, Marshall Shragg, Executive Director. Telephone: 612-548-2127, Toll Free 888-240-4762.

8. The Minneapolis Star Tribune, The St. Paul Pioneer Press, The Minnesota Dental Association News

9. A probable reason that "Specialists" did not out oppose the proposed Dental Therapist Legislation was because they foresaw that the Dental Therapist would be incapable of performing anything more than minimal restorative services, and they as "specialists" would then surely get more referrals.

10. ADA policy statement on evidence-based dentistry.

11. Ismail AI, Bader JD; ADA Council on Scientific Affairs and Division of Science Journal of the American Dental Association (2004) Evidence-based dentistry in clinica practice. J Am Dent Assoc 135: 78-83. [Crossref]

Copyright: $\odot 2015$ Wallin LA. This is an open-access article distributed under the terms of the Creative Commons Attribution License, which permits unrestricted use, distribution, and reproduction in any medium, provided the original author and source are credited. 